\title{
Eficacia de la ventilación no invasiva para la prevención de la reintubación
}

En los enfermos con un riesgo elevado de reintubación la aplicación profiláctica de ventilación no invasiva tras la extubación, comparada con el tratamiento médico estándar, reduce la frecuencia de reintubación, pero no modifica la mortalidad, ni la estancia hospitalaria.

In patients qith high risk of reintubation non-invasive ventilation is more effective than standard medical treatment in preventing reintubation, but it does not modify mortality, ICU, neither hospital mortality.

Artículo: Nava S, Gregoretti C, Farfulla F, Squadrone E, Grassi M, Carlucci A, et al. Noninvasive ventilation to prevent respiratory failure after extubation in high-risk patients. Crit Care Med. 2005;33:2465-70.

Antecedentes: la insuficiencia respiratoria postextubación es una complicación relativamente frecuente. Varios estudios han estimado su incidencia entre un $4 \%$ y un $24 \%$. En estudios no aleatorizados se ha observado que en los enfermos que desarrollan insuficiencia respiratoria hipercápnica postextubación la aplicación precoz de ventilación no invasiva (VNI) reduce la necesidad de reintubación. Sin embargo, dos ensayos clínicos aleatorizados, con $81^{1}$ y $221^{2}$ enfermos, que valoraron la eficacia de la VNI para tratar la insuficiencia respiratoria que aparece en las 48 horas posteriores a la extubación, no mostraron ningún beneficio de la VNI para reducir la frecuencia de reintubación, ni la mortalidad. Un ensayo clínico ${ }^{3}$ con asignación aleatoria evaluó la eficacia de la VNI (parámetros iniciales presión positiva espiratoria final [PEEP] $5 \mathrm{~cm} \mathrm{H} \mathrm{H}_{2} \mathrm{O}$; presión de soporte $12 \mathrm{~cm}$ $\mathrm{H}_{2} \mathrm{O}$ ) frente al tratamiento médico estándar para prevenir la reintubación en 93 enfermos de Unidades de Cuidados Intensivos [UCI] no seleccionados (56 tras extubación programada y 37 tras extubación accidental). La incidencia de reintubación fue $27,7 \%$ en el grupo tratado con VNI y $15,2 \%$ en el grupo tratado de modo estándar.
Objetivo: estimar si en los enfermos con alto riesgo de reintubación el uso preventivo de la VNI en las primeras 48 horas tras la extubación reduce la necesidad de reintubación.

Diseño: ensayo clínico con asignación aleatoria.

Período de estudio: no consta.

Lugar: tres Unidades de Cuidados Intensivos en Italia con experiencia en el uso de VNI.

Pacientes (tabla 1 y figura 1 ): Criterios de Inclusión: ventilación mecánica $>48$ horas que han tolerado una prueba de respiración espontánea durante 1 hora y que cumplan $\geq 1$ de los siguientes factores relacionados con una alta probabilidad de reintubación: a) fracaso en más de una prueba de respiración espontánea antes de la extubación; b) insuficiencia cardíaca; c) $\mathrm{PaCO}_{2}>45 \mathrm{mmHg}$ en la gasometría realizada 1 hora tras la extubación; $d$ ) más de una comorbilidad (excluyendo la insuficiencia cardíaca); e) tos débil definida como un Airway Care Score $\geq 8$ y $<12$ (tabla 2);f) signos de obstrucción de la vía aérea superior que no requiere reintubación inmediata.

Criterios de exclusión: 1. Coma. 2. Incapacidad para una adecuada protección de la vía aérea definida como Airway Care Score $\geq 12^{4}$. 
Tabla 1: Características generales

\begin{tabular}{|c|c|c|}
\hline & $\begin{array}{l}\text { Grupo Ventilación no invasiva } \\
\qquad(\mathrm{n}=49)\end{array}$ & $\begin{array}{l}\text { Grupo tratamiento médico estándar } \\
\qquad(\mathrm{n}=48)\end{array}$ \\
\hline Edad, años, media & 56 & 53 \\
\hline Género, mujeres, \% & 35 & 39 \\
\hline SAPS II, puntos, media & 31,4 & 32,5 \\
\hline \multicolumn{3}{|l|}{ Motivo para el inicio de la ventilación mecánica, \% } \\
\hline Enfermedad pulmonar obstructiva crónica & 36 & 31 \\
\hline Neumonía & 17 & 18 \\
\hline Síndrome de distrés respiratorio agudo & 13 & 10 \\
\hline Insuficiencia respiratoria aguda postoperatoria & 8 & 8 \\
\hline Traumatismo & 8 & 8 \\
\hline Insuficiencia cardíaca congestiva & 8 & 12 \\
\hline Neurocirugía & 6 & 11 \\
\hline \multicolumn{3}{|l|}{ Características en el momento de la asignación aleatoria } \\
\hline Duración de la intubación, días, media & 6 & 7 \\
\hline $\mathrm{PaCO}_{2}, \mathrm{mmHg}$, media & 42 & 39 \\
\hline Relación $\mathrm{PaO}_{2} / \mathrm{F}_{\mathrm{i}} \mathrm{O}_{2}, \mathrm{mmHg}$, media & 247 & 269 \\
\hline \multicolumn{3}{|l|}{ Factor de riesgo para reintubación, \% } \\
\hline $\mathrm{PaCO}_{2}>45$ mmHg (1hora tras extubación) & 37 & 31 \\
\hline Más de una co-morbilidad (exc. insuficiencia cardiaca) & 37 & 20 \\
\hline Más de una prueba de destete & 19 & 22 \\
\hline Insuficiencia cardiaca & 8 & 12 \\
\hline Tos débil & 4 & 6 \\
\hline Signos de obstrucción de la vía aérea superior & 4 & 2 \\
\hline
\end{tabular}

3. Problemas para la deglución. 4. Lesión en la columna cervical. 5. Enfermedad neuromucular. 6. Falta de consentimiento informado. 7. Agitación o falta de cooperación. 8. Defectos anatómicos que interfieren con la fijación de la máscara. 9. Arritmias o isquemia cardiaca no controladas. 10. Fracaso de más de dos órganos. 11. Obesidad mórbida (índice de masa corporal $\geq 30$ ). 12. Síndrome de apnea del sueño. 13. Ventilación mecánica domiciliaria.
Intervenciones que se comparan: una hora después de la extubación se obtuvo una gasometría arterial y los pacientes fueron asignados a:

Grupo ventilación no invasiva: los pacientes fueron ventilados con un ventilador específico para VNI (BiPAP Vision, Respironics) o con un ventilador convencional aplicando presión de soporte y PEEP extrínseca.

En los pacientes con hipercapnia la presión de soporte se ajustó de acuerdo con

Figura 1. Diagrama de inclusión

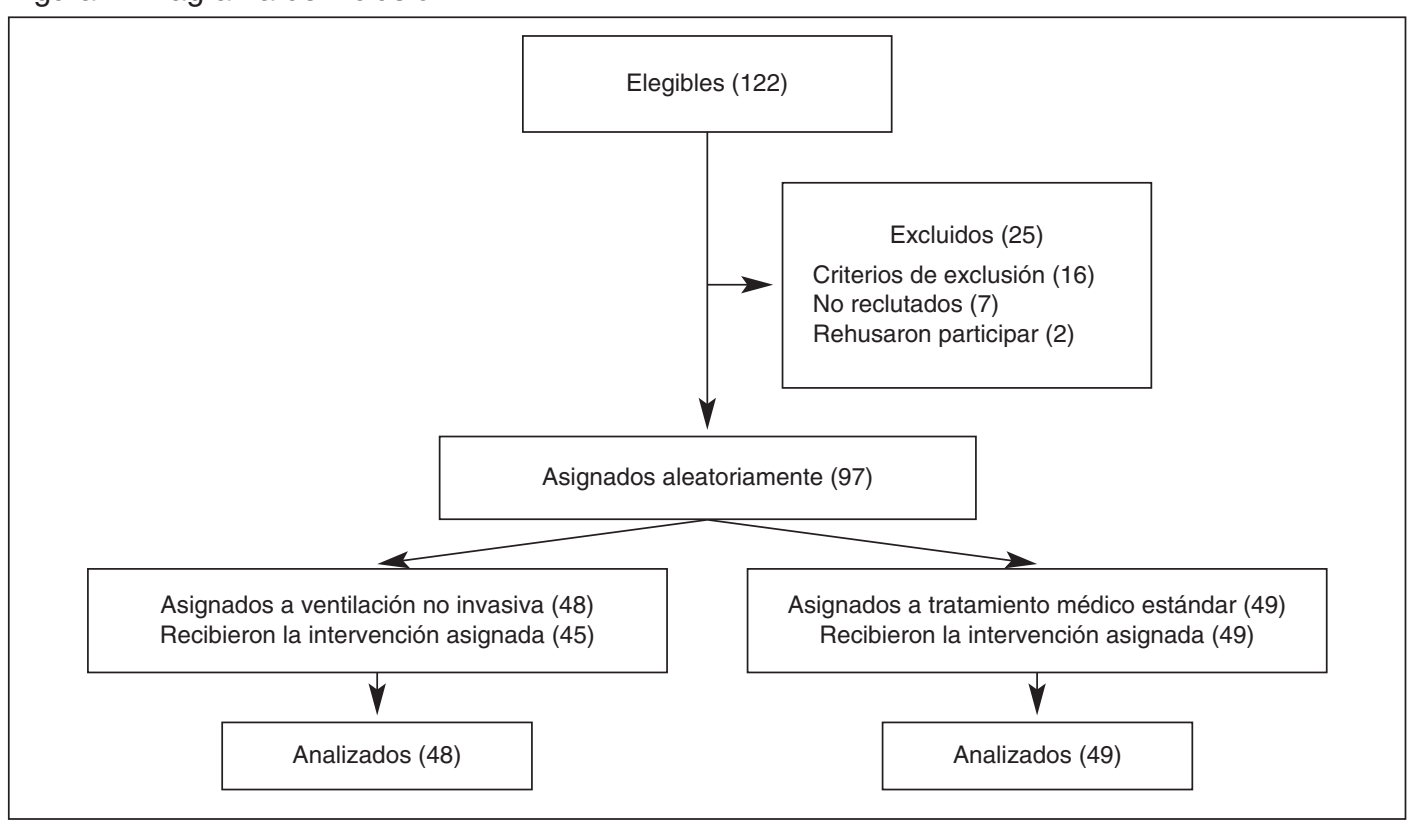


Tabla 2: Airway Care Score

\begin{tabular}{|c|c|c|c|c|c|c|}
\hline Puntuación & $\begin{array}{c}\text { Tos } \\
\text { espontánea }\end{array}$ & $\begin{array}{l}\text { Reflejo } \\
\text { tusígeno }\end{array}$ & $\begin{array}{l}\text { Cantidad } \\
\text { de esputo }\end{array}$ & $\begin{array}{l}\text { Viscosidad } \\
\text { del esputo }\end{array}$ & $\begin{array}{l}\text { Frecuencia } \\
\text { de aspiración } \\
\text { en un turno } \\
\text { de } 8 \text { horas }\end{array}$ & $\begin{array}{c}\text { Color } \\
\text { del esputo }\end{array}$ \\
\hline 0 & Vigorosa & Vigoroso & Nada & Acuosa & $>3 \mathrm{~h}$ & Claro \\
\hline 1 & Moderada & Moderado & 1 sonda de aspiración & Espumosa & Cada $2-3 \mathrm{~h}$ & Teñido \\
\hline 2 & Débil & Débil & 2 sondas de aspiración & Espesa & Cada $1-2 \mathrm{~h}$ & Amarillo \\
\hline 3 & Ninguna & Ninguno & $\geq 3$ sondas de aspiración & Muy espesa & $<1 \mathrm{~h}$ & Verde \\
\hline
\end{tabular}

La puntuación del Airway Care Score tiene un rango entre 0 y 18 puntos. Una puntuación alta es indicativa de una peor protección espontánea de la vía aérea.

Tabla 3: Necesidad de reintubación

\begin{tabular}{|lc|}
\hline Grupo ventilación no invasiva & $4 / 48(8 \%)$ \\
Grupo tratamiento médico estándar & $12 / 49(24 \%)$ \\
RR (IC 95\%) & $0,34(0,12$ a 0,98$)$ \\
RRR (IC 95\%) & $66 \%(7 \%$ a $125 \%)$ \\
RAR (IC 95\%) & $16 \%(2 \%$ a $31 \%)$ \\
NNB (IC 95\%) & $6(3$ a 56$)$ \\
\hline
\end{tabular}

RR: riesgo relativo; RAR: reducción absoluta riesgo; RRR: reducción relativa riesgo; NNB: número necesario para beneficiar; IC: intervalos de confianza.

la tolerancia del paciente. La PEEP extrínseca fue $<6 \mathrm{cmH}_{2} \mathrm{O}$.

En los pacientes sin hipercapnia, la PEEP extrínseca se programó, inicialmente, en $5 \mathrm{~cm}$ $\mathrm{H}_{2} \mathrm{O}$ y podía ser aumentada hasta obtener una $\mathrm{SaO}_{2}>92 \%$. La presión de soporte se programó, inicialmente, en $10 \mathrm{cmH}_{2} \mathrm{O}$ y se incrementó hasta el máximo tolerado. En ambos casos el objetivo fue mantener una frecuencia respiratoria $<25 \mathrm{resp} / \mathrm{min}$ $\mathrm{y}$ un adecuado intercambio gaseoso, definido como $\mathrm{SaO}_{2}>92 \%$ y $\mathrm{pH}>7,35$. La fracción inspirada de oxígeno fue la mínima necesaria para una $\mathrm{SaO}_{2}>92 \%$.

En las primeras 48 horas la VNI fue aplicada según un protocolo secuencial que consistía en la aplicación de presión de soporte al menos durante 30 minutos cada 3 horas, con un mínimo de 8 horas/día. Tras las primeras 48 horas, si el enfermo estaba estable, la ventilación no invasiva se retiraba, en otros casos se mantenía ad libitum.

Grupo tratamiento médico estándar: se administró oxigenoterapia para $\mathrm{SaO}_{2}>92 \%$.

El tratamiento médico estándar fue el que determinaron los médicos encargados del enfermo.

Asignación aleatoria: sí, oculta.
Enmascaramiento: no. Los desenlaces no fueron evaluados por un comité independiente.

Desenlaces principales: necesidad de reintubación.

Para ser reintubados debían cumplir $\geq 1$ criterio mayor $0, \geq 2$ criterios menores Los Criterios Mayores de reintubación fueron: a) acidosis respiratoria ( $\mathrm{pH}<7,35$ con una $\mathrm{PaCO}_{2}>45 \mathrm{mmHg}$ o, en el caso de hipercapnia tras la extubación, un aumento $\geq 15 \% ; b)$ hipoxemia $\left(\mathrm{SaO}_{2}<90 \%\right.$ con $\mathrm{FiO}_{2}$ $>50 \%$ ).

Los Criterios Menores de reintubación fueron: a) aumento $>20 \%$ de la frecuencia respiratoria desde el momento de la extubación y, en cualquier caso, frecuencia respiratoria $>35$ resp./min; $b$ ) signos clínicos de fatiga respiratoria (utilización de músculos accesorios, respiración abdominal paradójica); $c)$ disnea grave; $d$ ) incapacidad para expulsar secreciones (Airway Care Score > 12).

\section{Análisis por intención de tratar: sí.}

Resultados principales (tabla 3): el estudio fue interrumpido antes de alcanzar el tamaño muestral calculado porque en un análisis interino se cumplió la regla estadística prefijada para parar el ensayo clínico. Aunque no hubo diferencias significativas $(\mathrm{p}=0,06)$ en la mortalidad entre los dos grupo sí se observó una tendencia a favor de la VNI: $6 \%$ frente a $18 \%$.

La estancia en la UCI, la mortalidad hospitalaria y la estancia hospitalaria no mostraron diferencias entre ambos grupos.

Información sobre costes: no consta.

Financiación del estudio: no consta. 
Conclusiones de los autores: la VNI fue más efectiva que el tratamiento médico estándar en prevenir la insuficiencia respiratoria post-extubación en una población considerada de alto riesgo de desarrollar esta complicación.

Conclusiones de los revisores: los resultados del estudio muestran que la VNI es más eficaz como prevención de la insuficiencia respiratoria post-extubación que como tratamiento una vez esta complicación se ha desarrollado ${ }^{1,2}$. No obstante los resultados deben valorarse con cautela. Los criterios elegidos por los autores para determinar si un paciente tiene alto riesgo de reintubación no han sido apropiadamente validados. Posiblemente el uso de estos criterios permita seleccionar un grupo de enfermos con mayor riesgo de reintubación, ya que en este ensayo la necesidad de reintubación en el grupo con tratamiento estándar es significativamente mayor que la inciencia de reintubación publicada en estudios que incluyeron a todos los enfermos ventilados mecánicamente. No obstante, todavía no se ha obtenido una regla que prediga apropiadamente la necesidad de reintubación, lo que permitiría una mejor selección de enfermos en los que la VNI fuera eficaz. Los criterios gasométricos utilizados para la reintubación $\left(\mathrm{pH}<7,35\right.$ con $\mathrm{PaCO}_{2}>45 \mathrm{mmHg}$ o un aumento en la $\mathrm{PaCO}_{2}>15 \%$ respecto del nivel post-extubación) podrían haber perjudicado a los enfermos con EPOC agudizada ya que esta elevación de la $\mathrm{PaCO}_{2}$ llevó a la mayor parte de los enfermos con EPOC a valores de $\mathrm{pH} \leq 7,30$, en los que la VNI se ha mostrado útil para reducir la necesidad de intubación y la mortalidad. De hecho de los 5 con enfermos diagnosticados con EPOC que necesitaron reintubación 4 fueron tratados con tratamiento estándar y 1 con VNI. Por último conviene llamar la atención sobre la decisión de interrumpir los ensayos clínicos que muestran aparentemente beneficio de un tratamiento basada únicamente en criterios estadísticos. En general, los tamaños muestrales resultantes suelen ser pequeños, lo que implica potencialmente una distribución no similar entre los grupos de las variables asociadas a los desenlaces que se desean medir, y una imprecisión en la estimación del efecto evaluado, como ocurre en este estudio. Incluso los autores de la regla estadística utilizada en este ensayo clínico han llamado la atención reiteradamente sobre la insuficiencia, y las limitaciones éticas, de utilizar esa única norma para tomar la decisión de interrumpir un ensayo clínicos.

Posiblemente, al igual que en otros ensayos clínicos que han evaluado la eficacia de la VNI, de los enfermos con los criterios de inclusión definidos en este estudio, sean los enfermos con EPOC agudizado los que potencialmente se beneficien de la aplicación preventiva de la VNI postextubación.

\section{Bibliografía}

1. Keenan SP, Powers C, McCormack DG, Block G. Noninvasive positive-pressure ventilation for postextubation respiratory distress. A randomized controlled trial. JAMA. 2002;287:3238-44. (Revisado Med Intensiva. 2003;27:193-204).

2. Esteban A, Frutos-Vivar F, Ferguson ND, Arabi Y, Apezteguia C, González M, et al. Noninvasive positive-pressure ventilation for respiratory failure after extubation. N Engl J Med. 2004;350:2452-60. (Revisado Med Intensiva. 2004;28:481-3).
3. Jiang JS, Kao SJ, Wang SN. Effect of early application of biphasic positive airway pressure on the outcome of extubation in ventilator weaning. Respirology. 1999;4:161-5.

4. Coplin WM, Pierson DJ, Cooley KD, Newell DW, Rubenfeld GD. Implications of extubation delay in brain-injured patients meeting standard weaning criteria. Am J Respir Crit Care Med. 2000;161:1530-6.

5. Pocock SJ. When (not) to stop a clinical trial for benefit. JAMA. 2005;294:2228-30. 